\title{
Constant Term Methods in the Theory of Tesler matrices and Macdonald Polynomial Operators
}

\author{
by \\ A. Garsia, J. Haglund and G. Xin
}

\section{ABSTRACT}

The Tesler matrices with hook sums $\left(a_{1}, a_{2}, \ldots, a_{n}\right)$ are non-negative integral upper triangular matrices, whose $i^{\text {th }}$ diagonal element plus the sum of the entries in the arm of its (french) hook minus the sum of the entries in its leg is equal to $a_{i}$ for all $i$. In a recent paper [6], the second author expressed the Hilbert series of the Diagonal Harmonic modules as a weighted sum of the family of Tesler matrices with hook weights $(1,1, \ldots, 1)$. In this paper we use the constant term algorithm developed by the third author to obtain a Macdonald polynomial interpretation of these weighted sum of Tesler matrices for arbitrary hook weights. In particular we also obtain new and illuminating proofs of the results in [6].

\section{Introduction}

Denoting by $\mathcal{U P}$ the collection of upper triangular matrices with non-negative integer entries, let us set for a given integral vector $\left(a_{1}, a_{2}, \ldots, a_{n}\right)$

$$
\mathcal{T}\left(a_{1}, a_{2}, \ldots, a_{n}\right)=\left\{A=\left\|a_{i, j}\right\|_{i, j=1}^{n} \in \mathcal{U P}: a_{s, s}+\sum_{j=s+1}^{n} a_{s, j}-\sum_{i=1}^{s-1} a_{i, s}=a_{s} \quad \forall \quad 1 \leq s \leq n\right\} .
$$

We will here and after refer to this as the collection of Tesler matrices with hook sums $\left(a_{1}, a_{2}, \ldots, a_{n}\right)$.

To present our results we need to use plethystic notation. Readers unfamiliar with this notation are urged to read at least the beginning of the first section where we include an introductory Macdonald polynomial "tool kit".

The modified Macdonald polynomials $\left\{\tilde{H}_{\mu}[X ; q, t)\right\}_{\mu}$ we work with here are the unique symmetric function basis which satisfies the two triangularity conditions

$$
\text { a) } \quad \tilde{H}_{\mu}=\sum_{\lambda \leq \mu} s_{\lambda}\left[\frac{X}{t-1}\right] a_{\lambda \mu}(q, t), \quad \text { b) } \quad \tilde{H}_{\mu}=\sum_{\lambda \geq \mu} s_{\lambda}\left[\frac{X}{1-q}\right] b_{\lambda \mu}(q, t),
$$

together with the normalizing condition

$$
\left.\tilde{H}_{\mu}\right|_{s_{n}}=1 \quad \text { for all } \mu \text {. }
$$

Setting for a given partition $\mu=\left(\mu_{1}, \mu_{2}, \ldots, \mu_{k}\right)$

$$
n(\mu)=\sum_{i=1}^{k}(i-1) \mu_{i},
$$

the operator $\nabla$ was defined in [1] by setting

$$
\left.\nabla \tilde{H}_{\mu}=T_{\mu} \tilde{H}_{\mu} \quad \text { (with } T_{\mu}=t^{n(\mu)} q^{n\left(\mu^{\prime}\right)}\right) .
$$

Now it was conjectured in [3] and proved in [7] that the Hilbert series of the Diagonal Harmonics modules is given by the polynomial

$$
\partial_{p_{1}}^{n} \nabla e_{n}
$$


With this notation the result in [6] may be expressed in the form

$$
\partial_{p_{1}}^{n} \nabla e_{n}=\left(-\frac{1}{M}\right)^{n} \sum_{A \in \mathcal{T}(1,1, \ldots, 1)} w t(A)
$$

where for convenience we set for $A=\left\|a_{i, j}\right\|_{i, j=1}^{n}$

$$
w t(A)=\prod_{i, j} w t\left(a_{i, j}\right), \quad w t(a)=\left\{\begin{array}{ll}
-M^{\frac{q^{a}-t^{a}}{q-t}} & \text { if } a>0 \\
1 & \text { if } a=0
\end{array}, \quad M=(1-t)(1-q) .\right.
$$

In the same paper $[6]$ it is also shown that

$$
\left.\partial_{p_{1}}^{n} \nabla^{m} e_{n}=\left(-\frac{1}{M}\right)^{n} \sum_{A \in \mathcal{T}(1, m, \ldots, m)} w t(A) \quad \text { (for all } m \geq 1\right) .
$$

Note further that, as long as the hook sums $\left(a_{1}, a_{2}, \ldots, a_{n}\right)$ are strictly positive, each row of a matrix $A \in \mathcal{T}\left(a_{1}, a_{2}, \ldots, a_{n}\right)$ has to have at least one positive element. This is quite evident from the definition in I.1. Thus, in view of I.6, in spite of the denominator factor $(-M)^{n}$, the expression

$$
P_{a_{1}, a_{2}, \ldots, a_{n}}(q, t)=\left(-\frac{1}{M}\right)^{n} \sum_{A \in \mathcal{T}\left(a_{1}, a_{2}, \ldots, a_{n}\right)} w t(A)
$$

will necessarily evaluate to a polynomial. Further experimentations revealed that when $a_{1} \leq a_{2} \leq \cdots \leq a_{n}$ the polynomial $P_{a_{1}, a_{2}, \ldots, a_{n}}(q, t)$ turns out to have non-negative integer coefficients. One of our identities may lead to a Hilbert Scheme proof of this non-negativity. More precisely, we obtain the following equality

$$
P_{a_{1}, a_{2}, \ldots, a_{n}}(q, t)=(-1)^{n} M \partial_{p_{1}} \nabla^{-a_{n}} \partial_{p_{1}} \nabla^{a_{n}-a_{n-1}} \cdots \partial_{p_{1}} \nabla^{a_{3}-a_{2}} \partial_{p_{1}} \nabla^{a_{2}-a_{1}} \partial_{p_{1}} \nabla^{a_{1}} p_{n+1}\left[\frac{X}{M}\right],
$$

which (as we shall see) is easily shown to contain both I.5 and I.7.

The proof of I.9 is based on two identities which are of interest in their own right. The first of these identities involves the plethystic operators $D_{a}$ introduced in [4], which may simply be defined by setting for any symmetric function $F[X]$

$$
D_{a} F[X]=\left.F\left[X+\frac{M}{z}\right] \Omega[-z X]\right|_{z^{a}} .
$$

This given, we will show in section 2 that

$$
P_{a_{1}, a_{2}, \ldots, a_{n}}(q, t)=\left.\left(-\frac{1}{M}\right)^{n} D_{a_{1}-1} D_{a_{2}-1} \cdots D_{a_{n}-1} e_{n+1}\left[\frac{X}{M}\right]\right|_{X \rightarrow M} .
$$

In addition, also in section 2, we will give a new proof of I.5 as well as a proof of the surprising identity

$$
\left(-\frac{1}{M}\right)^{n-1} \sum_{A \in \mathcal{T}(n-1,-1,-1, \ldots,-1)} w t(A)=\left(-\frac{1}{M}\right)^{n-1} D_{-1}^{n} p_{n}\left[\frac{X}{M}\right]=\partial_{p_{1}}^{n-1} \nabla e_{n-1} .
$$

We should note that both these two results will be derived by manipulations involving only identities from our "tool kit". 
Section 3 will be dedicated to the proof of a truly remarkable recursive algorithm for the construction of the polynomials $P_{a_{1}, a_{2}, \ldots, a_{n}}(q, t)$. To state this result we need notation.

To begin, for any indeterminate $z$ let us set

$$
f(z)=\frac{(1-z)(1-z q t)}{(1-z t)(1-z q)}
$$

Next let $\mathcal{T}_{n}$ be the set of all Standard Young tableaux with labels $0,1,2, \ldots, n$. For a given $T \in \mathcal{T}_{n}$, we let $w_{T}(k)=q^{j-1} t^{i-1}$ if the label $k$ of $T$ is in the $i$-th row $j$-th column. We also denote by $S_{T}$ the substitution set $\left\{z_{k}^{-1}=w_{T}(k): 1 \leq k \leq n\right\}$. For instance, for $T=\left(\begin{array}{ccc}1 & 4 & \\ 0 & 2 & 3\end{array}\right)$ we have

$$
S_{T}=\left\{z_{1}^{-1}=t, z_{2}^{-1}=q, z_{3}^{-1}=q^{2}, z_{4}^{-1}=q t\right\} .
$$

This given, it will be shown in section 3 that

$$
P_{a_{1}, a_{2}, \ldots, a_{n}}(q, t)=\left.\sum_{T \in \mathcal{T}_{n}} \mathcal{H}_{n} \prod_{1 \leq k \leq n}\left(1-z_{k} w_{T}(k)\right) z_{k}^{-a_{k}}\right|_{S_{T}}
$$

where

$$
\mathcal{H}_{n}=\left(-\frac{1}{M}\right)^{n} \prod_{1 \leq i \leq n} f\left(z_{i}\right) \prod_{1 \leq i<j \leq n} f\left(z_{j} / z_{i}\right) .
$$

We must emphasize that I.14 is not a formula but rather an algorithm, since it should be understood that each summand must be separately constructed by progressive applications of the substitutions in the set $S_{T}$, and in the successive order $k=1,2, \ldots n$. This provision non withstanding we will show that the combination of I.11 and I.14 proves I.9.

\section{A Macdonald Polynomial tool kit}

The space of symmetric polynomials will be denoted $\Lambda$. The subspace of homogeneous symmetric polynomials of degree $m$ will be denoted by $\Lambda^{=m}$. We will seldom work with symmetric polynomials expressed in terms of variables but rather express them in terms of one of the six classical symmetric function bases

(1) "power" $\left\{p_{\mu}\right\}_{\mu}$, (2) "monomial" $\left\{m_{\mu}\right\}_{\mu}$, (3) "homogeneous" $\left\{h_{\mu}\right\}_{\mu}$,

(4) "elementary" $\left\{e_{\mu}\right\}_{\mu}$, (5) "forgotten" $\left\{f_{\mu}\right\}_{\mu}$ and (6) "Schur" $\left\{s_{\mu}\right\}_{\mu}$.

We recall that the fundamental involution $\omega$ may be defined by setting for the power basis indexed by $\mu=\left(\mu_{1}, \mu_{2}, \ldots, \mu_{k}\right) \vdash n$

$$
\omega p_{\mu}=(-1)^{n-k} p_{\mu}=(-1)^{|\mu|-l(\mu)} p_{\mu}
$$

where for any vector $v=\left(v_{1}, v_{2}, \cdots, v_{k}\right)$ we set $|v|=\sum_{i=1}^{k} v_{i}$ and $l(v)=k$.

In dealing with symmetric function identities, specially with those arising in the Theory of Macdonald Polynomials, we find it convenient and often indispensable to use plethystic notation. This device has a straightforward definition which can be verbatim implemented in MAPLE or MATHEMATICA for computer experimentation. We simply set for any expression $E=E\left(t_{1}, t_{2}, \ldots\right)$ and any power symmetric function $p_{k}$

$$
p_{k}[E]=E\left(t_{1}^{k}, t_{2}^{k}, \ldots\right) .
$$


This given, for any symmetric function $F$ we set

$$
F[E]=\left.Q_{F}\left(p_{1}, p_{2}, \ldots\right)\right|_{p_{k} \rightarrow E\left(t_{1}^{k}, t_{2}^{k}, \ldots\right)},
$$

where $Q_{F}$ is the polynomial yielding the expansion of $F$ in terms of the power basis. Note that in writing $E\left(t_{1}, t_{2}, \ldots\right)$ we are tacitly assuming that $t_{1}, t_{2}, t_{3}, \ldots$ are all the variables appearing in $E$ and in writing $E\left(t_{1}^{k}, t_{2}^{k}, \ldots\right)$ we intend that all the variables appearing in $E$ have been raised to their $k^{\text {th }}$ power.

A paradoxical but necessary property of plethystic substitutions is that 1.2 requires

$$
p_{k}[-E]=-p_{k}[E]
$$

This notwithstanding, we will still need to carry out ordinary changes of signs. To distinguish it from the "plethystic" minus sign, we will carry out the "ordinary" sign change by prepending our expressions with a superscripted minus sign, or as the case may be, by means of a new variables $\epsilon$ which outside of the plethystic bracket is simply replaced by -1 . For instance, these conventions give for $X_{n}=x_{1}+x_{2}+\cdots+x_{n}$

$$
p_{k}\left[-{ }^{-} X_{n}\right]=(-1)^{k-1} \sum_{i=1}^{n} x_{i}^{k},
$$

or, equivalently

$$
p_{k}\left[-\epsilon X_{n}\right]=-\epsilon^{k} \sum_{i=1}^{n} x_{i}^{k}=(-1)^{k-1} \sum_{i=1}^{n} x_{i}^{k} .
$$

In particular we get for $X=x_{1}+x_{2}+x_{3}+\cdots$

$$
\omega p_{k}[X]=p_{k}\left[-^{-} X\right]
$$

Thus for any symmetric function $F \in \Lambda$ and any expression $E$ we have

$$
\omega F[E]=F\left[-^{-} E\right]=F[-\epsilon E] .
$$

In particular, if $F \in \Lambda^{=k}$ we may also rewrite this as

$$
\left.F[-E]=\omega F^{-} E\right]=(-1)^{k} \omega F[E] .
$$

The formal power series

$$
\Omega=\exp \left(\sum_{k \geq 1} \frac{p_{k}}{k}\right)
$$

combined with plethystic substitutions will provide a powerful way of dealing with the many generating functions occurring in our manipulations.

Let us recall that in I.10 we have set

$$
D_{k} F[X]=\left.F\left[X+\frac{M}{z}\right] \Omega[-z X]\right|_{z^{k}} \quad \text { for }-\infty<k<+\infty
$$


with

$$
\Omega[-z X]=\sum_{m \geq 0} z^{m} h_{m}[-X]=\sum_{m \geq 0}(-1)^{m} z^{m} e_{m}[X] .
$$

Here " $\left.\right|_{z^{k}}$ " denotes the operation of taking the coefficient of $z^{k}$ in the preceding expression, $e_{m}$ and $h_{m}$ denote the elementary and homogeneous symmetric functions indexed by $m$.

To present our Macdonald polynomial kit, it is convenient to identify partitions with their (french) Ferrers diagram. Given a partition $\mu$ and a cell $c \in \mu$, Macdonald introduces four parameters $l=l_{\mu}(c), l^{\prime}=l_{\mu}^{\prime}(c), a=a_{\mu}(c)$ and $a^{\prime}=a_{\mu}^{\prime}(c)$ called leg, coleg, arm and coarm which give the number of lattice cells of $\mu$ strictly NORTH, SOUTH, EAST and WEST of $c$, (see attached figure). Following Macdonald we will set

$$
n(\mu)=\sum_{c \in \mu} l_{\mu}(c)=\sum_{c \in \mu} l_{\mu}^{\prime}(c)=\sum_{i=1}^{l(\mu)}(i-1) \mu_{i} .
$$

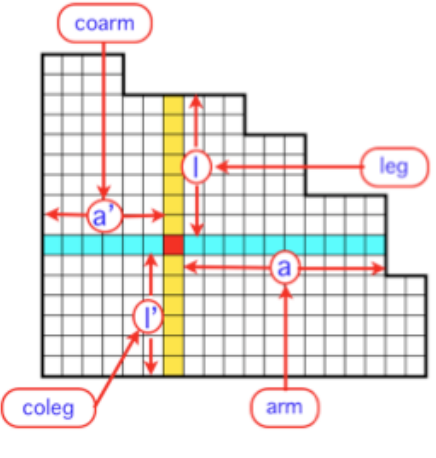

Denoting by $\mu^{\prime}$ the conjugate of $\mu$, the basic ingredients playing a role in the theory of Macdonald polynomials are

$$
\begin{gathered}
T_{\mu}=t^{n(\mu)} q^{n\left(\mu^{\prime}\right)}, \quad B_{\mu}(q, t)=\sum_{c \in \mu} t^{l_{\mu}^{\prime}(c)} q^{a_{\mu}^{\prime}(c)}, \quad \prod_{\mu}(q, t)=\prod_{c \in \mu ; c \neq(0,0)}\left(1-t^{l_{\mu}^{\prime}(c)} q^{a_{\mu}^{\prime}(c)}\right), \\
w_{\mu}(q, t)=\prod_{c \in \mu}\left(q^{a_{\mu}(c)}-t^{l_{\mu}(c)+1}\right)\left(t^{l_{\mu}(c)}-q^{a_{\mu}(c)+1}\right),
\end{gathered}
$$

together with a deformation of the Hall scalar product, which we call the "star" scalar product, defined by setting for the power basis

$$
\left\langle p_{\lambda}, p_{\mu}\right\rangle_{*}=(-1)^{|\mu|-l(\mu)} \prod_{i}\left(1-t^{\mu_{i}}\right)\left(1-q^{\mu_{i}}\right) z_{\mu} \chi(\lambda=\mu),
$$

where $z_{\mu}$ gives the order of the stabilizer of a permutation with cycle structure $\mu$.

The operators in 1.8 are connected to $\nabla$ and the polynomials $\tilde{H}_{\mu}$ through the following basic identities:

$$
\begin{array}{cc}
\text { (i) } & D_{0} \tilde{H}_{\mu}=-D_{\mu}(q, t) \tilde{H}_{\mu} \\
(\text { ii }) & D_{k} \underline{e}_{1}-\underline{e}_{1} D_{k}=M D_{k+1} \\
\text { (iii) } & \nabla \underline{e}_{1} \nabla^{-1}=-D_{1} \\
\text { (iv }) & \nabla^{-1} \partial_{1} \nabla=\frac{1}{M} D_{-1} \\
\text { (v) } & D_{k} \partial_{1}-\partial_{1} D_{k}=D_{k-1}
\end{array}
$$

where $\underline{e}_{1}$ is simply the operator "multiplication by $e_{1}$ ", and $\partial_{1}$ denotes its "Hall" scalar product adjoint.

Recall that for our version of the Macdonald polynomials the Macdonald Reciprocity formula states that

$$
\left.\frac{\tilde{H}_{\alpha}\left[1+u D_{\beta}\right]}{\prod_{c \in \alpha}\left(1-u t^{l^{\prime}} q^{a^{\prime}}\right)}=\frac{\tilde{H}_{\beta}\left[1+u D_{\alpha}\right]}{\prod_{c \in \beta}\left(1-u t^{l^{\prime}} q^{a^{\prime}}\right)} \quad \text { (for all pairs } \alpha, \beta\right)
$$

We will use here several special evaluations of 1.12. To begin, canceling the common factor $(1-u)$ out of the denominators on both sides of 1.12 and then setting $u=1$ gives

$$
\left.\frac{\tilde{H}_{\alpha}\left[M B_{\beta}\right]}{\Pi_{\alpha}}=\frac{\tilde{H}_{\beta}\left[M B_{\alpha}\right]}{\Pi_{\beta}} \quad \text { (for all pairs } \alpha, \beta\right) .
$$


On the other hand replacing $u$ by $1 / u$ and letting $u=0$ in 1.12 gives

$$
\left.(-1)^{|\alpha|} \frac{\tilde{H}_{\alpha}\left[D_{\beta}\right]}{T_{\alpha}}=(-1)^{|\beta|} \frac{\tilde{H}_{\beta}\left[D_{\alpha}\right]}{T_{\beta}} \quad \text { (for all pairs } \alpha, \beta\right) .
$$

Since for $\beta$ the empty partition we can take $\tilde{H}_{\beta}=1$ and $D_{\beta}=-1,1.12$ in this case reduces to

$$
\tilde{H}_{\alpha}[1-u]=\prod_{c \in \alpha}\left(1-u t^{l^{\prime}} q^{a^{\prime}}\right)=(1-u) \sum_{r=0}^{n-1}(-u)^{r} e_{r}\left[B_{\mu}-1\right] .
$$

This identity yields the coefficients of hook Schur functions in the expansion.

$$
\tilde{H}_{\mu}[X ; q, t]=\sum_{\lambda \vdash|\mu|} s_{\mu}[X] \tilde{K}_{\lambda \mu}(q, t) .
$$

Recall that the addition formula for Schur functions gives

$$
s_{\mu}[1-u]= \begin{cases}(-u)^{r}(1-u) & \text { if } \mu=\left(n-r, 1^{r}\right) \\ 0 & \text { otherwise }\end{cases}
$$

Thus 1.16, with $X=1-u$, combined with 1.15 gives for $\mu \vdash n$

$$
\left\langle\tilde{H}_{\mu}, s_{\left(n-r, 1^{r}\right)}\right\rangle=e_{r}\left[B_{\mu}-1\right]
$$

and the identity $e_{r} h_{n-r}=s_{\left(n-r, 1^{r}\right)}+s_{\left(n-r-1,1^{r-1}\right)}$ gives

$$
\left\langle\tilde{H}_{\mu}, e_{r} h_{n-r}\right\rangle=e_{r}\left[B_{\mu}\right] .
$$

Since for $\beta=(1)$ we have $\tilde{H}_{\beta}=1$ and $\Pi_{\beta}=1$, formula 1.13 reduces to the surprisingly simple identity

$$
\tilde{H}_{\alpha}[M]=M B_{\alpha} \Pi_{\alpha} .
$$

Last but not least we must also recall that we have the Pieri formulas

$$
\text { a) } e_{1} \tilde{H}_{\nu}=\sum_{\mu \leftarrow \nu} d_{\mu \nu} \tilde{H}_{\mu}, \quad \text { b) } e_{1}^{\perp} \tilde{H}_{\mu}=\sum_{\nu \rightarrow \mu} c_{\mu \nu} \tilde{H}_{\nu}
$$

Here $\nu \rightarrow \mu$ simply means that the sum is over $\nu$ 's obtained from $\mu$ by removing a corner cell and $\mu \leftarrow \nu$ means that the sum is over $\mu$ 's obtained from $\nu$ by adding a corner cell.

The final ingredient we need, to carry out our proofs are expressions, proved in [11], for the coefficients $d_{\mu, \nu}$ in terms of the corner weights of the partition $\mu$. More precisely, let $\mu$ have $m$ outer corners $A_{0}, A_{1}, \ldots, A_{m}$ (see adjacent figure) and inner corners $B_{1}, B_{2}, \ldots, B_{m}$. Then the weight of $A_{i}$ is $x_{i}=t^{\alpha_{i+1}} q^{\beta_{i}}$ with $\alpha_{i}$ and $\beta_{i}$ the coleg and coarm of $B_{i}$ in $\mu$. The weight of $B_{i}$ will then be $u_{i}=t^{\alpha_{i}} q^{\beta_{i}}$. It is also convenient to set $\beta_{0}=0$ and $\alpha_{m+1}=0$.

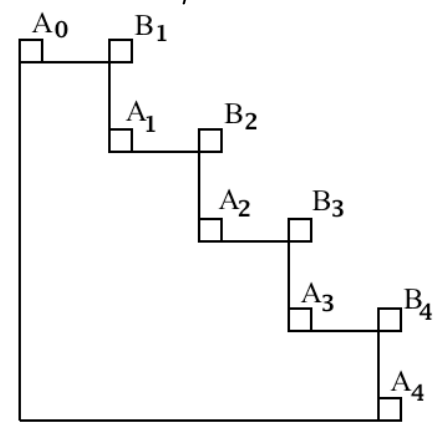


This given, in the proof of I.9 we will make use of the following two identities

$$
x_{0}+x_{1}+\cdots+x_{m}-u_{1}-\cdots-u_{m}=-M B_{\mu}(q, t)+1
$$

and

$$
d_{\mu^{i} \nu}=\frac{1}{x_{i}} \frac{\prod_{j=1}^{m}\left(1-u_{j} / x_{i}\right)}{\prod_{j=0, j \neq i}^{m}\left(1-x_{j} / x_{i}\right)}
$$

where $\mu^{i}$ is the partition obtained by adding to $\nu$ corner $A_{i}$. The proof of both identities is given in [11]. We must note that 1.22 follows easily from the geometry of the above diagram, while 1.23 results from massive cancellations occurring in the original Macdonald Pieri formulas.

It will also be useful to know that these two Pieri coefficients are related by the identity

$$
d_{\mu \nu}=M c_{\mu \nu} \frac{w_{\nu}}{w_{\mu}}
$$

Recall that our Macdonald Polynomials satisfy the orthogonality condition

$$
\left\langle\tilde{H}_{\lambda}, \tilde{H}_{\mu}\right\rangle_{*}=\chi(\lambda=\mu) w_{\mu}(q, t) .
$$

The *-scalar product, is simply related to the ordinary Hall scalar product by setting for all pairs of symmetric functions $f, g$

$$
\langle f, g\rangle_{*}=\langle f, \omega \phi g\rangle,
$$

where it has been customary to let $\phi$ be the operator defined by setting for any symmetric function $f$

$$
\phi f[X]=f[M X]
$$

Note that the inverse of $\phi$ is usually written in the form

$$
f^{*}[X]=f[X / M] .
$$

In particular we also have for all symmetric functions $f, g$

$$
\langle f, g\rangle=\left\langle f, \omega g^{*}\right\rangle_{*}
$$

The orthogonality relations in 1.25 yield the "Cauchy" identity for our Macdonald polynomials in the form

$$
\Omega\left[-\epsilon \frac{X Y}{M}\right]=\sum_{\mu} \frac{\tilde{H}_{\mu}[X] \tilde{H}_{\mu}[Y]}{w_{\mu}},
$$

which restricted to its homogeneous component of degree $n$ in $X$ and $Y$ reduces to

$$
e_{n}\left[\frac{X Y}{M}\right]=\sum_{\mu \vdash n} \frac{\tilde{H}_{\mu}[X] \tilde{H}_{\mu}[Y]}{w_{\mu}} .
$$

Note that the orthogonality relations in 1.25 yield us the following Macdonald polynomial expansions 


\section{Proposition 1.1}

For all $n \geq 1$ we have

$$
\begin{aligned}
& \text { a) } e_{n}\left[\frac{X}{M}\right]=\sum_{\mu \vdash n} \frac{\tilde{H}_{\mu}[X]}{w_{\mu}}, \quad \text { b) } h_{k}\left[\frac{X}{M}\right] e_{n-k}\left[\frac{X}{M}\right]=\sum_{\mu \vdash n} \frac{e_{k}\left[B_{\mu}\right] \tilde{H}_{\mu}[X]}{w_{\mu}}, \quad \text { c) } h_{n}\left[\frac{X}{M}\right]=\sum_{\mu \vdash n} \frac{T_{\mu} \tilde{H}_{\mu}[X]}{w_{\mu}} \\
& \text { d) }(-1)^{n-1} p_{n}=\left(1-t^{n}\right)\left(1-q^{n}\right) \sum_{\mu \vdash n} \frac{\Pi_{\mu} \tilde{H}_{\mu}[X]}{w_{\mu}} \\
& \text { e) } \quad e_{1}[X / M]^{n}=\sum_{\mu \vdash n} \frac{\tilde{H}_{\mu}[X]}{w_{\mu}}\left\langle\tilde{H}_{\mu}, e_{1}^{n}\right\rangle \\
& \text { f) } \quad e_{n}=\sum_{\mu \vdash m} \frac{\tilde{H}_{\mu}[X] M B_{\mu} \Pi_{\mu}}{w_{\mu}}
\end{aligned}
$$

Finally it is good to keep in mind, for future use, that we have for all partitions $\mu$

$$
T_{\mu} \omega \tilde{H}_{\mu}[X ; 1 / q, 1 / t]=\tilde{H}_{\mu}[X ; q, t]
$$

\section{Remark 1.1}

It was conjectured in [3] and proved in [7] that the bigraded Frobenius characteristic of the diagonal Harmonics of $S_{n}$ is given by the symmetric function

$$
D H_{n}[X ; q, t]=\sum_{\mu \vdash n} \frac{T_{\mu} \tilde{H}_{\mu}(X ; q, t) M B_{\mu}(q, t) \Pi_{\mu}(q, t)}{w_{\mu}(q, t)} .
$$

Surprisingly the intricate rational function on the right hand side is none other than $\nabla e_{n}$. To see this we simply combine the relation in 1.20 with the degree $n$ restricted Macdonald-Cauchy formula 1.31 obtaining

$$
e_{n}[X]=e_{n}\left[\frac{X M}{M}\right]=\sum_{\mu \vdash n} \frac{\tilde{H}_{\mu}[X] M B_{\mu} \Pi_{\mu}}{w_{\mu}} .
$$

This is perhaps the simplest way to prove $1.32 \mathrm{f}$ ). This discovery is precisely what led to the introduction of $\nabla$ in the first place.

\section{Iterated plethystic operators identities}

Our first goal here is to obtain our new proof of I.5 based on the connection between Tesler matrices and plethystic operators. The basic ingredient in this approach is provided by the following

\section{Proposition 2.1}

For any symmetric function $F[X]$ and any sequence of integers $a_{1}, a_{2}, \ldots, a_{n}$ we have

$$
\begin{aligned}
\left.D_{a_{n}} \cdots D_{a_{2}} D_{a_{1}} F[X]\right|_{X=M} & =\left.F\left[M+\sum_{i=1}^{n} \frac{M}{z_{i}}\right] \prod_{i=1}^{n} \Omega\left[-z_{i} M\right] \prod_{1 \leq i<j \leq n} \Omega\left[-\frac{z_{i}}{z_{j}} M\right]\right|_{z_{1}^{a_{1}} z_{2}^{a_{2} \ldots z_{n}^{a_{n}}}} \\
& =\left.F\left[M+\sum_{i=1}^{n} \frac{M}{z_{i}}\right] \prod_{i=1}^{n} \frac{\left(1-z_{i}\right)\left(1-t q z_{i}\right)}{\left(1-t z_{i}\right)\left(1-q z_{i}\right)} \prod_{1 \leq i<j \leq n} \frac{\left(1-\frac{z_{i}}{z_{j}}\right)\left(1-t q \frac{z_{i}}{z_{j}}\right)}{\left(1-t \frac{z_{i}}{z_{j}}\right)\left(1-q \frac{z_{i}}{z_{j}}\right)}\right|_{z_{1}^{a_{1}} z_{2}^{a_{2} \cdots z_{n}^{a}}}
\end{aligned}
$$




\section{Proof}

The definition in 1.8 gives

$$
D_{a_{1}} F[X]=\left.F\left[X+\frac{M}{z_{1}}\right] \Omega\left[-z_{1} X\right]\right|_{z_{1}^{a_{1}}}
$$

and using 1.8 again we get

$$
\begin{aligned}
D_{a_{2}} D_{a_{1}} F[X] & =\left.F\left[X+\frac{M}{z_{1}}+\frac{M}{z_{2}}\right] \Omega\left[-z_{1}\left(X+\frac{M}{z_{2}}\right)\right] \Omega\left[-z_{2} X\right]\right|_{z_{1}^{a_{1}} z_{2}^{a_{2}}} \\
& \left.=F\left[X+\frac{M}{z_{1}}+\frac{M}{z_{2}}\right] \Omega\left[-\frac{z_{1}}{z_{2}} M\right] \Omega\left[-z_{1} X\right]\right]\left.\Omega\left[-z_{2} X\right]\right|_{z_{1}^{a_{1}} z_{2}^{a_{2}}}
\end{aligned}
$$

This given, it is easy to see that the successive actions of $D_{a_{3}} \cdots D_{a_{k}}$ will eventually yield the identity

$$
D_{a_{n}} \cdots D_{a_{2}} D_{a_{1}} F[X]=\left.F\left[X+\sum_{i=1}^{n} \frac{M}{z_{i}}\right] \prod_{1 \leq i<j \leq n} \Omega\left[-\frac{z_{i}}{z_{j}} M\right] \prod_{i=1}^{n} \Omega\left[-z_{i} X\right]\right|_{z_{1}^{a_{1}} z_{2}^{a_{2} \ldots z_{n}^{a_{n}}}} .
$$

Setting $X=M$ gives the first equality in 1.6, but then the second equality holds as well since for any indeterminate $v$ we have

$$
\Omega[-v M]=\Omega[v t+v q-v-q t v]=\frac{(1-v)(1-q t v)}{(1-t v)(1-q v)} .
$$

Two immediate corollaries of Proposition 2.1 are identities I.11 and I.12:

\section{Theorem 2.1}

$$
\begin{gathered}
P_{a_{1}, a_{2}, \ldots, a_{n}}(q, t)=\left.\left(-\frac{1}{M}\right)^{n} D_{a_{1}-1} D_{a_{2}-1} \cdots D_{a_{n}-1} e_{n+1}\left[\frac{X}{M}\right]\right|_{X \rightarrow M} \\
\left(-\frac{1}{M}\right)^{n-1} \sum_{A \in \mathcal{T}(n-1,-1,-1, \ldots,-1)} w t[A]=\left(-\frac{1}{M}\right)^{n-1} D_{-1}^{n} p_{n}\left[\frac{X}{M}\right]=\partial_{p_{1}}^{n-1} \nabla e_{n-1}
\end{gathered}
$$

\section{Proof}

Setting $F[X]=e_{n+1}\left[\frac{X}{M}\right]$ the second equality in 2.1 gives

$$
\begin{aligned}
\left.D_{a_{n}} \cdots D_{a_{2}} D_{a_{1}} F[X]\right|_{X=M} & =\left.e_{n+1}\left[1+\sum_{i=1}^{n} \frac{1}{z_{i}}\right] \prod_{i=1}^{n} \Omega\left[-z_{i} M\right] \prod_{1 \leq i<j \leq n} \Omega\left[-\frac{z_{i}}{z_{j}} M\right]\right|_{z_{1}^{a_{1}} z_{2}^{a_{2} \ldots z_{n}}} \\
& =\left.\prod_{i=1}^{n} \Omega\left[-z_{i} M\right] \prod_{1 \leq i<j \leq n} \Omega\left[-\frac{z_{i}}{z_{j}} M\right]\right|_{z_{1}^{a_{1}+1} z_{2}^{a_{2}+1} \cdots z_{n}^{a_{n}+1}},
\end{aligned}
$$

where the second equality is due to the identity

$$
e_{n+1}\left[1+\sum_{i=1}^{n} \frac{1}{z_{i}}\right]=\frac{1}{z_{1} z_{2} \cdots z_{n}} .
$$

Now note that the generic summand produced by the expression

$$
H\left(b_{1}, b_{2}, \ldots, b_{n}\right)=\left.\prod_{i=1}^{n} \Omega\left[-z_{i} M\right] \prod_{1 \leq i<j \leq n} \Omega\left[-\frac{z_{i}}{z_{j}} M\right]\right|_{z_{1}^{b_{1}} z_{2}^{b_{2}} \ldots z_{n}^{b_{n}}}
$$


after we expand all its factors according to 1.9 is

$$
\left.\prod_{i=1}^{n} z_{i}^{p_{i}} h_{p_{i}}[-M] \prod_{1 \leq i<j \leq n}\left(z_{i} / z_{j}\right)^{p_{i j}} h_{p_{i j}}[-M]\right|_{z_{1}^{b_{1}} z_{2}^{b_{2}} \cdots z_{n}^{b_{n}}}
$$

This forces the equalities

$$
p_{s}+\sum_{j=s+1}^{n} p_{s j}-\sum_{i=1}^{s-1} p_{i s}=b_{s} \quad(\text { for all } 1 \leq s \leq n)
$$

We may thus associate to each summand an upper triangular matrix $A=\left\|a_{i, j}\right\|_{i, j=1}^{n}$ by setting

$$
a_{i, j}= \begin{cases}p_{i} & \text { if } i=j \\ p_{i j} & \text { if } i<j \\ 0 & \text { otherwise }\end{cases}
$$

Since it can be shown that

$$
h_{a}[-M]= \begin{cases}-M \frac{q^{a}-t^{a}}{q-t} & \text { if } a \geq 1 \\ 1 & \text { if } a=0\end{cases}
$$

we immediately derive from this observation, and the definition in I.8 that

$$
\left.\prod_{i=1}^{n} \Omega\left[-z_{i} M\right] \prod_{1 \leq i<j \leq n} \Omega\left[-\frac{z_{i}}{z_{j}} M\right]\right|_{z_{1}^{b_{1}} z_{2}^{b_{2}} \cdots z_{n}^{b_{n}}}=\sum_{A \in T\left(b_{1}, b_{2}, \ldots, b_{n}\right)} w t(A) .
$$

Thus 2.3 follows from 2.5 with the replacements $a_{i} \rightarrow a_{i}-1$.

To prove 2.4 we start with the definition of $D_{-1}$ and get

$$
D_{-1} p_{n}\left[\frac{X}{M}\right]=\left.\left(p_{n}\left[\frac{X}{M}\right]+\frac{1}{z^{n}}\right) \Omega[-z X]\right|_{z^{-1}}=(-1)^{n-1} e_{n-1}
$$

Using this and 1.11 (iv) we derive that

$$
\left(-\frac{1}{M}\right)^{n-1} D_{-1}^{n} p_{n}\left[\frac{X}{M}\right]=\left(\frac{1}{M}\right)^{n-1} D_{-1}^{n-1} e_{n-1}=\nabla^{-1} \partial_{p_{1}}^{n-1} \nabla e_{n-1}=\partial_{p_{1}}^{n-1} \nabla e_{n-1}
$$

On the other hand Proposition 2.1 with $a_{1}=a_{2}=\cdots=a_{n}=-1$ and $F[X]=p_{n}\left[\frac{X}{M}\right]$ gives

$$
\left.D_{-1}^{n} p_{n}\left[\frac{X}{M}\right]\right|_{X=M}=\left.\left(1+\sum_{i=1}^{n} \frac{1}{z_{i}^{n}}\right) \prod_{i=1}^{n} \Omega\left[-z_{i} M\right] \prod_{1 \leq i<j \leq n} \Omega\left[-\frac{z_{i}}{z_{j}} M\right]\right|_{z_{1}^{-1} z_{2}^{-1} \cdots z_{n}^{-1}}
$$

and 2.9 gives

$$
\partial_{p_{1}}^{n-1} \nabla e_{n-1}=\left.\left(-\frac{1}{M}\right)^{n-1}\left(1+\sum_{i=1}^{n} \frac{1}{z_{i}^{n}}\right) \prod_{i=1}^{n} \Omega\left[-z_{i} M\right] \prod_{1 \leq i<j \leq n} \Omega\left[-\frac{z_{i}}{z_{j}} M\right]\right|_{z_{1}^{-1} z_{2}^{-1} \cdots z_{n}^{-1}}
$$

Note next that if we set $F=1$ and $a_{1}=a_{2}=\cdots=a_{n}=-1$ in 2.1 we obtain

$$
0=\left.D_{-1}^{n} 1\right|_{X=M}=\left.\prod_{i=1}^{n} \Omega\left[-z_{i} M\right] \prod_{1 \leq i<j \leq n} \Omega\left[-\frac{z_{i}}{z_{j}} M\right]\right|_{z_{1}^{-1} z_{2}^{-1} \cdots z_{n}^{-1}}
$$


that eliminates one of the terms in 2.10. We claim that the only term that survives in 2.10 is the one yielded by $\frac{1}{z_{1}^{n}}$. That is we have

$$
\left.\left.\frac{1}{z_{k}^{n}} \prod_{i=1}^{n} \Omega\left[-M z_{i}\right] \prod_{1 \leq i<j \leq n} \Omega\left[-M z_{i} / z_{j}\right]\right|_{z_{1}^{-1} z_{2}^{-1} \cdots z_{n}^{-1}}=0 \quad \text { (for all } 2 \leq k \leq n\right) .
$$

To see this let us rewrite the LHS in the expanded form, that is

$$
\left.\frac{1}{z_{k}^{n}} \prod_{i=1}^{n} \sum_{p_{i} \geq 0} z_{i}^{p_{i}} h_{p_{i}}[-M] \prod_{1 \leq i<j \leq n} \sum_{r_{i, j} \geq 0} \frac{z_{i}^{r_{i, j}}}{z_{j}^{r_{i, j}}} h_{r_{i, j}}[-M]\right|_{z_{1}^{-1} z_{2}^{-1} \cdots z_{n}^{-1}} .
$$

The exponent of $z_{1}$ in the generic term of the product of these geometric series must satisfy the equation

$$
p_{1}+\sum_{j=2}^{n} r_{1, j}=-1 .
$$

This is, of course impossible, causing 2.13 to be true precisely as asserted.

Now using 2.13 and 2.12 in 2.11 gives

$$
\partial_{p_{1}}^{n-1} \nabla e_{n-1}=\left.\left(-\frac{1}{M}\right)^{n-1} \prod_{i=1}^{n} \Omega\left[-z_{i} M\right] \prod_{1 \leq i<j \leq n} \Omega\left[-\frac{z_{i}}{z_{j}} M\right]\right|_{z_{1}^{n-1} z_{2}^{-1} \cdots z_{n}^{-1}}
$$

and this, combined with 2.8 and 2.9 proves that

$$
\partial_{p_{1}}^{n-1} \nabla e_{n-1}=\left(-\frac{1}{M}\right)^{n-1} D_{-1}^{n} p_{n}\left[\frac{X}{M}\right]=\left(-\frac{1}{M}\right)^{n-1} \sum_{A \in \mathcal{T}(n-1,-1,-1, \ldots,-1)} w_{H}[A]
$$

and our proof is complete.

Our proof of I.5 is more elaborate and requires the following auxiliary identity.

\section{Proposition 2.2}

For any symmetric function $F[X]$ we have

$$
\sum_{\mu \vdash n+1} \frac{M B_{\mu} \Pi_{\mu}}{w_{\mu}} F\left[M B_{\mu}\right]=\left.\Delta_{e_{n}} F[X]\right|_{X=M} .
$$

Proof

We need only prove this for $F[X]=\tilde{H}_{\gamma}[X]$ for arbitrary $\gamma$. In this case 2.15 becomes

$$
\sum_{\mu \vdash n+1} \frac{M B_{\mu} \Pi_{\mu}}{w_{\mu}} \tilde{H}_{\gamma}\left[M B_{\mu}\right]=\tilde{H}_{\gamma}[M] e_{n}\left[B_{\gamma}\right]=M B_{\gamma} \Pi_{\gamma} e_{n}\left[B_{\gamma}\right] .
$$

Since by the reciprocity in 1.13 we have

$$
\frac{\tilde{H}_{\gamma}\left[M B_{\mu}\right]}{\Pi_{\gamma}}=\frac{\tilde{H}_{\mu}\left[M B_{\gamma}\right]}{\Pi_{\mu}}
$$


2.16 becomes

$$
\Pi_{\gamma} \sum_{\mu \vdash n+1} \frac{M B_{\mu}}{w_{\mu}} \tilde{H}_{\mu}\left[M B_{\gamma}\right]=M B_{\gamma} \Pi_{\gamma} e_{n}\left[B_{\gamma}\right]
$$

Or better

$$
\sum_{\mu \vdash n+1} \frac{M B_{\mu}}{w_{\mu}} \tilde{H}_{\mu}\left[M B_{\gamma}\right]=M B_{\gamma} e_{n}\left[B_{\gamma}\right]
$$

But recall that we have

$$
B_{\mu}=\sum_{\nu \rightarrow \mu} c_{\mu \nu}
$$

and 2.17 becomes

$$
\sum_{\mu \vdash n+1} \frac{M}{w_{\mu}} \tilde{H}_{\mu}\left[M B_{\gamma}\right] \sum_{\nu \rightarrow \mu} c_{\mu \nu}=M B_{\gamma} e_{n}\left[B_{\gamma}\right] .
$$

Now for the left hand side we have

$$
\begin{aligned}
L H S & =\sum_{\nu \vdash n} \frac{1}{w_{\nu}} \sum_{\mu \leftarrow \nu} \frac{M w_{\nu}}{w_{\mu}} c_{\mu \nu} \tilde{H}_{\mu}\left[M B_{\gamma}\right] \\
(\text { by } 1.24) & =\sum_{\nu \vdash n} \frac{1}{w_{\nu}} \sum_{\mu \leftarrow \nu} d_{\mu \nu} \tilde{H}_{\mu}\left[M B_{\gamma}\right] \\
(\text { by } 1.21 \mathrm{a})) & =\sum_{\nu \vdash n} \frac{1}{w_{\nu}} e_{1}\left[M B_{\gamma}\right] \tilde{H}_{\nu}\left[M B_{\gamma}\right] \\
(\text { by } 1.32 \mathrm{a})) & =e_{1}\left[M B_{\gamma}\right] e_{n}\left[\frac{M B_{\gamma}}{M}\right]=M B_{\gamma} e_{n}\left[B_{\gamma}\right]=R H S
\end{aligned}
$$

and our proof is complete.

As a corollary we obtain

\section{Proposition 2.3}

For $F \in \Lambda^{=k}$ with $k \leq n$ we have

$$
\sum_{\mu \vdash n+1} \frac{M B_{\mu} \Pi_{\mu}}{w_{\mu}} F\left[M B_{\mu}\right]= \begin{cases}\left.\nabla F[X]\right|_{X=M} & \text { if } k=n \\ 0 & \text { if } k<n\end{cases}
$$

\section{Proof}

From 2.15 we get that the left hand side of 2.19 is

$$
\left.\Delta_{e_{n}} F[X]\right|_{X=M}
$$

but for a symmetric function $F[X]$ of degree $n$ we have $\Delta_{e_{n}} F[X]=\nabla F[X]$. Thus the first alternative in 2.19 is immediate. On the other hand for $k<n$ the expansion of $F$ in the Macdonald basis will involve $\tilde{H}_{\gamma}^{\prime} s$ with $\gamma \vdash k$ and even before we make the evaluation at $X=M$ the identity

$$
\left.\Delta_{e_{n}} \tilde{H}_{\gamma}[X]=e_{n}\left[B_{\gamma}\right] \tilde{H}_{\gamma}[S]=0 \quad \text { (for all } \gamma \vdash k<n\right)
$$


forces $\Delta_{e_{n}} F=0$, yielding the second alternative in 2.19 .

We are now in a position to give our new and direct proof of I.5.

\section{Theorem 2.2}

$$
\partial_{p_{1}}^{n} \nabla e_{n}=\left.\left(-\frac{1}{M}\right)^{n} D_{0}^{n} e_{n+1}\left[\frac{X}{M}\right]\right|_{X=M} .
$$

Proof

From $1.32 \mathrm{a}$ ) and 1.11 (i) we get

$$
\begin{aligned}
\left.D_{0}^{n} e_{n+1}\left[\frac{X}{M}\right]\right|_{X=M}=\left.\left(D_{0}^{n} \sum_{\mu \vdash n+1} \frac{\tilde{H}_{\mu}[X ; q, t]}{w_{\mu}}\right)\right|_{X=M} & =\left.\sum_{\mu \vdash n+1} \frac{\tilde{H}_{\mu}[X ; q, t]\left(1-M B_{\mu}\right)^{n}}{w_{\mu}}\right|_{X=M} \\
& =\sum_{k=0}^{n}\left(\begin{array}{l}
n \\
k
\end{array}\right)(-M)^{k} \sum_{\mu \vdash n+1} \frac{M B_{\mu} \Pi_{\mu}}{w_{\mu}} B_{\mu}^{k}
\end{aligned}
$$

and Proposition 2.3 with $F=e_{1}\left[\frac{X}{M}\right]^{k}$ and $0 \leq k \leq n$ gives

$$
\begin{aligned}
\left.\left(-\frac{1}{M}\right)^{n} D_{0}^{n} e_{n+1}\left[\frac{X}{M}\right]\right|_{X=M} & =\left.\nabla e_{1}^{n}\left[\frac{X}{M}\right]\right|_{X=M} \\
\text { (by } 1.32 \text { e) and the definition of } \nabla) & \left.=\sum_{\mu \vdash n} \frac{T_{\mu} \tilde{H}_{\mu}[X]}{w_{\mu}}\left\langle\tilde{H}_{\mu}, e_{1}^{n}\right\rangle\right]\left.\right|_{X=M} \\
(\text { by } 1.20) & =\sum_{\mu \vdash n} \frac{T_{\mu} M B_{\mu} \Pi_{\mu}}{w_{\mu}}\left\langle\tilde{H}_{\mu}, e_{1}^{n}\right\rangle
\end{aligned}
$$

(by $1.32 \mathrm{f}$ ) and $\left.\partial_{p_{1}}=e_{1}^{\perp}\right)=\partial_{p_{1}}^{n} \nabla e_{n}$

This proves 2.20 and completes our argument.

\section{Constant terms and positivity of Tesler matrix polynomials}

Our first goal here is to establish I.9. It will be convenient to write it here in the form

$$
P_{a_{1}, a_{2}, \ldots, a_{n}}(q, t)=M \partial_{p_{1}}\left(\prod_{i=1}^{n} \nabla^{-a_{i}} \partial_{p_{1}} \nabla^{a_{i}}\right)(-1)^{n} p_{n+1}\left[\frac{X}{M}\right]
$$

provided it is understood that the operator factors are successively applied from right to left, starting with $\nabla^{-a_{n}} \partial_{p_{1}} \nabla^{a_{n}}$ and ending with $\nabla^{-a_{1}} \partial_{p_{1}} \nabla^{a_{1}}$.

Our starting point is a semi-combinatorial interpretation of the right hand side. To state it we need some notation. As in the introduction, we let $\mathcal{T}_{n}$ be the set of all Standard Young tableaux (SYTs for short) with labels $0,1,2, \ldots, n$. For a given $T \in \mathcal{T}_{n}$, we let $w_{T}(k)=q^{j-1} t^{i-1}$ if the label $k$ of $T$ is in the $i$-th row $j$-th column and let $\operatorname{sh}(T)$ denote the partition giving the shape of $T$. Recall that we have set

$$
S_{T}=\left\{z_{k}^{-1}=w_{T}(k): 1 \leq k \leq n\right\} .
$$

For a tableau $T \in \mathcal{T}_{n}$ we let $T^{(i)}$ denote the tableau in $\mathcal{T}_{i}$ obtained by removing $i+1, i+2, \ldots, n$ from $T$. It will be convenient to also let $T^{(n)}=T$. This given, for any $0 \leq i \leq n$ we set

$$
\begin{gathered}
c_{T^{(i)} / T^{(i-1)}}=c_{s h\left(T^{(i)}\right), \operatorname{sh}\left(T^{(i-1)}\right)}, \quad d_{T^{(i)} / T^{(i-1)}}=d_{s h\left(T^{(i)}\right), \operatorname{sh}\left(T^{(i-1)}\right)} \\
w_{T^{(i)}}=w_{s h\left(T^{(i)}\right)}, \quad B_{T^{(i)}}=B_{s h\left(T^{(i)}\right)}, \quad \Pi_{T^{(i)}}=\Pi_{s h\left(T^{(i)}\right)} .
\end{gathered}
$$


Note that if $\mu \vdash n+1$ then I.4 and $1.21 \mathrm{~b}$ ) give

$$
\nabla^{-a} \partial_{p_{1}} \nabla^{a} \tilde{H}_{\mu}=\sum_{\nu \rightarrow \mu} c_{\mu, \nu}\left(\frac{T_{\mu}}{T_{\nu}}\right)^{a} \tilde{H}_{\nu}
$$

Since the monomial $\frac{T_{\mu}}{T_{\nu}}$ is none other than the weight of the corner cell that we must remove from $\mu$ to get $\nu$. To avoid notational conflicts let us set, for a moment $\frac{T_{\mu}}{T_{\nu}}=w t(\mu / \nu)$. Now it is easily seen that $w t\left(\operatorname{sh}\left(T^{(k)}\right) / \operatorname{sh}\left(T^{(k-1)}\right)\right)$ is precisely the weight of the cell that contains $k$ in $T^{(k)}$, which was earlier denoted $w_{T}(k)$. Thus here in the following we can set

$$
w t\left(\operatorname{sh}\left(T^{(k)}\right) / \operatorname{sh}\left(T^{(k-1)}\right)\right)=w_{T}(k) .
$$

This given, we can now give the right hand side of 3.1 the following standard tableau expansion.

\section{Proposition 3.1}

$$
M \partial_{p_{1}}\left(\prod_{i=1}^{n} \nabla^{-a_{i}} \partial_{p_{1}} \nabla^{a_{i}}\right)(-1)^{n} p_{n+1}\left[\frac{X}{M}\right]=\frac{1}{M^{n}} \sum_{T \in \mathcal{T}_{n}} \prod_{k=1}^{n} w_{T}(k)^{a_{k}}\left(1-w_{T}(k)\right) d_{T^{(k)} / T^{(k-1)}}
$$

\section{Proof}

Using $1.32 \mathrm{~d}$ ) the left hand side of 3.6 becomes

$$
L H S=\sum_{\mu \vdash n+1} \frac{M \Pi_{\mu}}{w_{\mu}}\left(\partial_{p_{1}} \prod_{i=1}^{n} \nabla^{-a_{i}} \partial_{p_{1}} \nabla^{a_{i}}\right) \tilde{H}_{\mu}[X] .
$$

For $n=1$ we have for $\mu=(1,1)$ and $\mu=(2)$

$$
\partial_{p_{1}} \nabla^{-a_{1}} \partial_{p_{1}} \nabla^{a_{1}} \tilde{H}_{(1,1)}[X]=t^{a_{1}} c_{(1,1),(1)}, \quad \partial_{p_{1}} \nabla^{-a_{1}} \partial_{p_{1}} \nabla^{a_{1}} \tilde{H}_{(2)}[X]=q^{a_{1}} c_{(2),(1)} .
$$

Thus we can inductively assume that for $\nu \vdash n$

$$
\partial_{p_{1}}\left(\prod_{i=1}^{n-1} \nabla^{-a_{i}} \partial_{p_{1}} \nabla^{a_{i}}\right) \tilde{H}_{\nu}[X]=\sum_{T \in \mathcal{T}_{n-1} ; s h(T)=\nu} \prod_{k=1}^{n-1} w_{T}(k)^{a_{k}} c_{T^{(k)} / T^{(k-1)}} .
$$

But for $\mu \vdash n+1$ we have

$$
\nabla^{-a_{n}} \partial_{p_{n}} \nabla^{a_{1}} \tilde{H}_{\mu}[X]=\sum_{\nu \rightarrow \mu} w t(\mu / \nu)^{a_{n}} c_{\mu, \nu} \tilde{H}_{\nu}[X]
$$

and 3.8 gives

$$
\partial_{p_{1}}\left(\prod_{i=1}^{n} \nabla^{-a_{i}} \partial_{p_{1}} \nabla^{a_{i}}\right) \tilde{H}_{\mu}[X]=\sum_{\nu \rightarrow \mu} w t(\mu / \nu)^{a_{n}} c_{\mu, \nu} \sum_{T \in \mathcal{T}_{n-1} ; s h(T)=\nu} \prod_{k=1}^{n-1} w_{T}(k)^{a_{k}} c_{T^{(k)} / T^{(k-1)}} .
$$

Now this can clearly be rewritten as

$$
\partial_{p_{1}}\left(\prod_{i=1}^{n} \nabla^{-a_{i}} \partial_{p_{1}} \nabla^{a_{i}}\right) \tilde{H}_{\mu}[X]=\sum_{T \in \mathcal{T}_{n} ; s h(T)=\mu} \prod_{k=1}^{n} w_{T}(k)^{a_{k}} c_{T^{(k)} / T^{(k-1)}},
$$


completing the induction. Using 3.9 in 3.7 yields that

$$
\begin{aligned}
L H S & =\sum_{\mu \vdash n+1} \frac{M \Pi_{\mu}}{w_{\mu}} \sum_{T \in \mathcal{T}_{n} ; s h(T)=\mu} \prod_{k=1}^{n} w_{T}(k)^{a_{k}} c_{T^{(k)} / T^{(k-1)}} \\
& =\sum_{T \in \mathcal{T}_{n}} \Pi_{T} \frac{M}{w_{T}} \prod_{k=1}^{n} w_{T}(k)^{a_{k}} c_{T^{(k)} / T^{(k-1)}} .
\end{aligned}
$$

Since the definition in 1.10 gives $W_{(1)}=M$ we can write

$$
\Pi_{T} \frac{M}{w_{T}}=\prod_{k=1}^{n}\left(1-c w\left(T^{(k)}\right) \frac{w_{T^{(k-1)}}}{w_{T^{(k)}}}\right.
$$

and 3.10 becomes

$$
\begin{aligned}
L H S & =\sum_{T \in \mathcal{T}_{n}} \prod_{k=1}^{n} w_{T}(k)^{a_{k}}\left(1-c w\left(T^{(k)}\right) \frac{w_{T^{(k-1)}}}{w_{T^{(k)}}} c_{T^{(k)} / T^{(k-1)}}\right. \\
\text { (Using 1.24) } & =\frac{1}{M^{n}} \sum_{T \in \mathcal{T}_{n}} \prod_{k=1}^{n} w_{T}(k)^{a_{k}}\left(1-c w\left(T^{(k)}\right) d_{T^{(k)} / T^{(k-1)}},\right.
\end{aligned}
$$

completing our proof of 3.6 .

Now recall that we have shown in 2.8 that

$$
\sum_{A \in T\left(a_{n}, a_{n-1}, \ldots, a_{1}\right)} w t(A)=\left.\prod_{i=1}^{n} \frac{1}{z_{i}^{a_{i}}} \Omega\left[-z_{i} M\right] \prod_{1 \leq i<j \leq n} \Omega\left[-\frac{z_{j}}{z_{i}} M\right]\right|_{z_{1}^{0} z_{2}^{0} \cdots z_{n}^{0}}
$$

which 2.2 allows us to rewrite in the form

$$
\sum_{A \in T\left(a_{n}, a_{n-1}, \ldots, a_{1}\right)} w t(A)=\left.\prod_{i=1}^{n} \frac{1}{z_{i}^{a_{i}}} \frac{\left(1-z_{i}\right)\left(1-q t z_{i}\right)}{\left(1-t z_{i}\right)\left(1-q z_{i}\right)} \prod_{1 \leq i<j \leq n} \frac{\left(1-z_{j} / z_{i}\right)\left(1-q t z_{j} / z_{i}\right)}{\left(1-t z_{j} z_{i}\right)\left(1-q z_{j} / z_{i}\right)}\right|_{z_{1}^{0} z_{2}^{0} \cdots z_{n}^{0}} .
$$

Thus our proof of 3.1 will be complete by showing that

$$
\begin{gathered}
\left.\prod_{i=1}^{n} \frac{1}{z_{i}^{a_{i}}} \frac{\left(1-z_{i}\right)\left(1-q t z_{i}\right)}{\left(1-t z_{i}\right)\left(1-q z_{i}\right)} \prod_{1 \leq i<j \leq n} \frac{\left(1-z_{j} / z_{i}\right)\left(1-q t z_{j} z_{i}\right)}{\left(1-t z_{j} / z_{i}\right)\left(1-q z_{j} / z_{i}\right)}\right|_{z_{1}^{0} z_{2}^{0} \cdots z_{n}^{0}}= \\
=\sum_{T \in \mathcal{T}_{n}} \prod_{k=1}^{n} w_{T}(k)^{a_{k}}\left(1-w_{T}(k)\right) d_{T^{(k)} / T^{(k-1)}} .
\end{gathered}
$$

\section{Remark 3.1}

We will find it convenient to note that the factor $d_{T^{(k)} / T^{(k-1)}}$ and 3.13 itself can be given a revealing expression by manipulations with our " $\Omega$ " symbol. To see this we start by rewriting 1.23 in terms of $\Omega$ as follows 


$$
\begin{aligned}
d_{\mu^{(i)}, \nu} & =\frac{1}{x_{i}} \frac{\prod_{j=1}^{m}\left(1-u_{j} / x_{i}\right)}{\prod_{j=0, j \neq i}^{m}\left(1-x_{j} / x_{i}\right)}=\left.z \frac{\prod_{j=1}^{m}\left(1-z u_{j}\right)}{\prod_{j=0, j}^{m}\left(1-z x_{j}\right)}\left(1-z x_{i}\right)\right|_{z=1 / x_{i}} \\
& =\left.z \Omega\left[z\left(x_{0}+\cdots+x_{m}-u_{1}-\cdots-u_{m}\right)\right]\left(1-z x_{i}\right)\right|_{z=1 / x_{i}} .
\end{aligned}
$$

Taking account that $x_{i}$ is the weight of the cell that contains $k$ in $T^{(k)}$, using 1.22 we obtain

$$
\begin{aligned}
d_{T^{(k)} / T^{(k-1)}} & =\left.z \Omega\left[-z\left(M B_{T^{(k-1)}}-1\right)\right]\left(1-z w_{T}(k)\right)\right|_{1 / z=w_{T}(k)} \\
& =\left.z \Omega\left[-z M B_{T^{(k-1)}}+z-z w_{T}(k)\right]\right|_{1 / z=w_{T}(k)} \\
& \left.=\frac{1}{w_{T}(k)} \Omega\left[-\frac{1}{w_{T}(k)} M B_{T^{(k-1)}}+\frac{1}{w_{T}(k)}-1\right)\right] \\
& \left.=\frac{1}{w_{T}(k)\left(1-\frac{1}{w_{T}(k)}\right)} \Omega\left[-\frac{1}{w_{T}(k)} M B_{T^{(k-1)}}-1\right)\right] .
\end{aligned}
$$

Using 1.22 again this may be rewritten as

$$
\left.\left(1-w_{T}(k)\right) d_{T^{(k)} / T^{(k-1)}}=-\Omega\left[-\frac{1}{w_{T}(k)} M\left(1+w_{T}(1)+\cdots+w_{T}(k-1)\right)-1\right)\right] .
$$

Thus 3.13 becomes none other than

$$
\begin{aligned}
\left.\prod_{i=1}^{n} \frac{1}{z_{i}^{a_{i}}} \frac{\left(1-z_{i}\right)\left(1-q t z_{i}\right)}{\left(1-t z_{i}\right)\left(1-q z_{i}\right)} \prod_{1 \leq i<j \leq n} \frac{\left(1-z_{j} / z_{i}\right)\left(1-q t z_{j} / z_{i}\right)}{\left(1-t z_{j} / z_{i}\right)\left(1-q z_{j} / z_{i}\right)}\right|_{z_{1}^{0} z_{2}^{0} \cdots z_{n}^{0}}= \\
\left.\quad=\sum_{T \in \mathcal{T}_{n}}(-1)^{n} \prod_{k=1}^{n} w_{T}(k)^{a_{k}} \Omega\left[-\frac{1}{w_{T}(k)} M\left(1+w_{T}(1)+\cdots+w_{T}(k-1)\right)-1\right)\right] \\
\left.\quad=\sum_{T \in \mathcal{T}_{n}}(-1)^{n} \prod_{k=1}^{n} w_{T}(k)^{a_{k}} \Omega\left[-M / w_{T}(k)\right] \prod_{k=1}^{n} \Omega\left[-M\left(\sum_{h=1}^{k-1} w_{T}(h) / w_{T}(k)\right)-1\right)\right] .
\end{aligned}
$$

In summary, the proof of 3.1 is thus reduced to verify the constant term identity

$$
\begin{aligned}
\left.\frac{1}{M^{n}} \prod_{i=1}^{n} \frac{1}{z_{i}^{a_{i}}} \frac{\left(1-z_{i}\right)\left(1-q t z_{i}\right)}{\left(1-t z_{i}\right)\left(1-q z_{i}\right)} \prod_{1 \leq i<j \leq n} \frac{\left(1-z_{j} / z_{i}\right)\left(1-q t z_{j} / z_{i}\right)}{\left(1-t z_{j} / z_{i}\right)\left(1-q z_{j} / z_{i}\right)}\right|_{z_{1}^{0} z_{2}^{0} \cdots z_{n}^{0}}= \\
=\sum_{T \in \mathcal{T}_{n}}\left(-\frac{1}{M}\right)^{n} \prod_{k=1}^{n} w_{T}(k)^{a_{k}} \Omega\left[-M \sum_{1 \leq k \leq n} w_{T}(k)^{-1}-M \sum_{1 \leq h<k \leq n} w_{T}(h) / w_{T}(k)-n\right] .
\end{aligned}
$$

This will be done by means of the partial fraction algorithm developed in [9]. To benefit the reader who is unfamiliar with this algorithm we will briefly review here its basic steps.

Firstly, to avoid ordinary convergence problems we need to work in the field of iterated formal Laurent series. The definition of this field is recursive and is determined by a chosen total order of all the variables appearing in our given "kernel" $\mathcal{H}$. In the applications we are to compute the constant term of $\mathcal{H}$, usually denoted $\mathcal{H}_{=0}$. To be precise this operation will involve only a specific subset of the variables. For simplicity let us assume this subset to be $z_{1}, z_{2}, \ldots, z_{k}$, and here we use the notation $\left.\mathcal{H}\right|_{z_{1}^{0} z_{2}^{0} \cdots z_{k}^{0}}$. 
The first operation consists in expanding $\mathcal{H}$ as a formal iterated Laurent series and selecting the terms that do not contain any of the variables $z_{1}, z_{2}, \ldots z_{k}$. This is done by a succession of a single variable constant term extractions.

Supposing that our variables, in the chosen total order, are $z_{1}, z_{2}, \ldots, z_{n}$. Then, for a given field of scalars $K$ the initial field is $K\left(\left(z_{1}\right)\right)$ consisting of formal Laurent series in $z_{1}$ with coefficients in $K$, that is the series in which $z_{1}$ appears with a negative exponent only in a finite number of terms. In symbols

$$
K\left(\left(z_{1}\right)\right)=\left\{\sum_{m \geq M_{0}} a_{m} z_{1}^{m}: a_{m} \in K\right\} .
$$

This given, recursively we define the field of iterated Laurent series $K\left(\left(z_{1}\right)\right)\left(\left(z_{2}\right)\right) \cdots\left(\left(z_{n}\right)\right)$ to be the field of formal Laurent series in $z_{n}$ with coefficients in $K\left(\left(z_{1}\right)\right)\left(\left(z_{2}\right)\right) \cdots\left(\left(z_{n-1}\right)\right)$. The fundamental fact is that the total order allows us to imbed the field of rational functions $K\left(z_{1}, z_{2}, \ldots, z_{n}\right)$ as a subfield of $K\left(\left(z_{1}\right)\right)\left(\left(z_{2}\right)\right) \cdots\left(\left(z_{n}\right)\right)$. We shall only describe here how this imbedding is carried out but leave all the matters of consistency to the original works [9], [10]. The important fact is that under this imbedding all the identities in $K\left(z_{1}, z_{2}, \ldots, z_{n}\right)$ become identities in $K\left(\left(z_{1}\right)\right)\left(\left(z_{2}\right)\right) \cdots\left(\left(z_{n}\right)\right)$.

We will begin with the recipe for converting each rational function in the given variables into a formal Laurent series. The rational functions we will work with here may all be written in the form

$$
F=\frac{P}{\left(1-m_{1}\right)\left(1-m_{2}\right) \cdots\left(1-m_{\ell}\right)}
$$

with $P$ a Laurent polynomial and $m_{1}, m_{2}, \ldots, m_{\ell}$ monomials in the given variables. Our first need is to be able to decide whether a given factor $\frac{1}{1-m_{i}}$ should be converted to

$$
\text { a) } \left.\sum_{s \geq 0} m_{i}^{s} \quad \text { or } \quad b\right) \quad-\sum_{s \geq 1} \frac{1}{m_{i}^{s}} \quad\left(=\frac{-\frac{1}{m_{1}}}{1-\frac{1}{m_{1}}}\right)
$$

The decision is based on the idea that the total order forces one of the two "formal" inequalities $m_{i}<1$ or $m_{i}>1$ to be true. In the first case, we choose a) (the "ordinary form") and in the second case, we choose b) (the "dual form"). The criterion is as follows: we scan through the variables occurring in the monomial $m_{i}$. Suppose $m_{i}$ has the variable $z_{j}$ but not $z_{j+1}, \ldots, z_{n}$. Then $m_{i}<1$ if $z_{j}$ has positive exponent and $m_{i}>1$ if $z_{j}$ has negative exponent.

For simplicity of notation we will avoid using summations and simply rewrite the given rational function in the form

$$
F=P \times\left(\prod_{m_{i}<1} \frac{1}{1-m_{i}}\right) \times\left(\prod_{m_{j}>1} \frac{-\frac{1}{m_{j}}}{1-\frac{1}{m_{j}}}\right) .
$$

We shall refer to this symbolic expression as the "proper form" of $F$.

To compute $\left.F\right|_{z_{1}^{0} z_{2}^{0} \cdots z_{k}^{0}}$ by the partial fraction algorithm, at each step we use a partial fraction expansion to eliminate one of the variables $z_{1}, z_{2}, \ldots, z_{k}$.

To see how this is done, assume that to begin we have chosen to eliminate the variable $z$. This given, by suitable manipulations we rewrite our rational function in the form

$$
F=Q(z)+\frac{R(z)}{\left(1-z U_{1}\right) \cdots\left(1-z U_{h}\right)\left(z-V_{1}\right) \cdots\left(z-V_{k}\right)}
$$


with $Q(z)$ a Laurent polynomial, $R(z)$ a polynomial of degree less than $h+k$ and $U_{1}, U_{2}, \ldots, U_{h}$ as well as $V_{1}, V_{2}, \ldots, V_{k}$ are monomials not containing $z$. The nature of the denominator will be determined by the requirement that

$$
z U_{i}<1 \quad \text { for } 1 \leq i \leq h \quad \text { and } \quad V_{j} / z<1 \quad \text { for } 1 \leq j \leq k .
$$

The next step is to derive the partial fraction expansion:

$$
F=Q(z)+\sum_{i=1}^{h} \frac{A_{i}}{\left(1-z U_{i}\right)}+\sum_{j=1}^{k} \frac{B_{j}}{\left(z-V_{j}\right)}
$$

which, as customary, is obtained by setting

$$
A_{i}=\left.\left(1-z U_{i}\right) F(z)\right|_{z=1 / U_{i}} \quad \text { and } \quad B_{j}=\left.\left(z-V_{j}\right) F(z)\right|_{z=V_{j}} .
$$

This immediately yields the equalities

$$
\left.F\right|_{z^{0}}=\left.Q(z)\right|_{z^{0}}+\sum_{i=1}^{h} A_{i}
$$

The reason for this is that $V_{j} / z<1$ by assumption, so that the proper form of the last summation in 3.17 will be

$$
\sum_{j=1}^{k} \frac{B_{j} / x}{\left(1-V_{j} / z\right)}
$$

and we see that the corresponding series contains only negative powers of $z$ and thus yields no contribution to $\left.F\right|_{z^{0}}$. For this reason we say that the denominator factors $\left(1-z U_{i}\right)$ are "contributing" and the factors $\left(z-V_{j}\right)$ are "not contributing".

Keeping all this in mind, we will proceed to establish, by these methods, an auxiliary constant term result which at the same time will yield us both I.14 and 3.15.

To begin, for convenience, since we plan to eliminate the variables $z_{1}, z_{2}, \ldots, z_{n}$ in their natural order, we will write our kernel in the form

$$
\mathcal{H}_{n}(z ; q, t)=\frac{1}{(-M)^{n}} \prod_{1 \leq i \leq n} f\left(z_{i}\right) \prod_{1 \leq i<j \leq n} f\left(z_{j} / z_{i}\right)
$$

with

$$
f(z)=\frac{(1-z)(1-q t z)}{(1-t z)(1-q z)}
$$

This given the auxiliary constant term result may be stated as follows. 


\section{Theorem}

Suppose $F_{n}(z)=\sum_{a_{i} \geq 1} c_{a_{1}, \ldots, a_{n}} z_{1}^{-a_{1}} \cdots z_{n}^{-a_{n}}$ contains only negative powers in the $z$ 's and $c_{a_{1}, \ldots, a_{n}}$ do not depend on the $z^{\prime} s$. Then

$$
\left.\mathcal{H}_{n}(z ; q, t) F_{n}(z)\right|_{z^{0}}=\left.\sum_{T \in \mathcal{T}_{n}} \mathcal{H}_{n}(z ; q, t) F_{n}(z) \prod_{1 \leq k \leq n}\left(1-z_{k} w_{T}(k)\right)\right|_{S_{T}} .
$$

Here it must be understood that the substitution $S_{T}$ has to be carried out iteratively. That means we first multiply by $\left(1-z_{1} w_{T}(1)\right)$ and make the substitution for $z_{1}$, then we multiply by $\left(1-z_{2} w_{T}(2)\right)$ and make the substitution for $z_{2}$, and so on, always keeping track of the cancellations that occur.

Proof

We will proceed by induction on $n$. For $n=1$ we need to compute the constant term

$$
\mathcal{H}_{1}(z ; q, t)=\left.\frac{1}{-M} \frac{\left(1-z_{1}\right)\left(1-q t z_{1}\right)}{\left(1-q z_{1}\right)\left(1-t z_{1}\right)} F_{1}(z)\right|_{z_{1}^{0}} .
$$

This is a proper rational function in $z_{1}$ and both denominator factors $\left(1-q z_{1}\right)$ and $\left(1-t z_{1}\right)$ are contributing, so the constant term is equal to

$$
\left.\mathcal{H}_{1}(z ; q, t) F_{1}(z) \cdot\left(1-z_{1} q\right)\right|_{z_{1}=q^{-1}}+\left.\mathcal{H}_{1}(z ; q, t) F_{1}(z) \cdot\left(1-z_{1} t\right)\right|_{z_{1}=t^{-1}} \cdot
$$

This agrees with the right hand side of 3.21 since

$$
\left.\mathcal{T}_{2}=\left\{\left[\begin{array}{l}
0 \\
1
\end{array}\right],\left[\begin{array}{ll}
0 & 1
\end{array}\right]\right\} \text {, and } S_{\left[\begin{array}{l}
0 \\
1
\end{array}\right]}=\left\{z_{1}^{-1}=t\right\}, S_{[0} 1\right]=\left\{z_{1}^{-1}=q\right\} .
$$

Now suppose the theorem holds for $n$ and we need to show that it holds for $n+1$. So we need to compute the constant term

$$
\begin{aligned}
\left.\mathcal{H}_{n+1}(z ; q, t) F_{n+1}(z)\right|_{z_{1}^{0} z_{2}^{0} \cdots z_{n+1}^{0}} & =\left.\prod_{1 \leq i \leq n+1} \frac{\left(1-z_{i}\right)\left(1-q t z_{i}\right)}{\left(1-q z_{i}\right)\left(1-t z_{i}\right)} \prod_{1 \leq i<j \leq n+1} \frac{\left(1-z_{j} / z_{i}\right)\left(1-q t z_{j} / z_{i}\right)}{\left(1-q z_{j} / z_{i}\right)\left(1-t z_{j} / z_{i}\right)} F_{n+1}(z)\right|_{z_{1}^{0} z_{2}^{0} \cdots z_{n+1}^{0}} \\
& =\left.\prod_{1 \leq i \leq n} \frac{\left(1-z_{i}\right)\left(1-q t z_{i}\right)}{\left(1-q z_{i}\right)\left(1-t z_{i}\right)} \prod_{1 \leq i<j \leq n} \frac{\left(1-z_{j} / z_{i}\right)\left(1-q t z_{j} / z_{i}\right)}{\left(1-q z_{j} / z_{i}\right)\left(1-t z_{j} / x_{i}\right)} F^{\prime}(z)\right|_{z_{1}^{0} z_{2}^{0} \cdots z_{n+1}^{0}} \\
& =\left.\mathcal{H}_{n}(z ; q, t) F^{\prime}(z)\right|_{z_{1}^{0} z_{2}^{0} \cdots z_{n+1}^{0}}
\end{aligned}
$$

where

$$
F^{\prime}(z)=F_{n+1}(z) \frac{\left(1-z_{n+1}\right)\left(1-q t z_{n+1}\right)}{\left(1-q z_{n+1}\right)\left(1-t z_{n+1}\right)} \prod_{1 \leq i \leq n} \frac{\left(1-z_{n+1} / z_{i}\right)\left(1-q t z_{n+1} / z_{i}\right)}{\left(1-q z_{n+1} / z_{i}\right)\left(1-t z_{n+1} / z_{i}\right)}
$$

contains only negative powers in $z_{i}$ for $i=1,2, \ldots, n$. Thus the inductive hypothesis applies to the variables $z_{1}, \ldots, z_{n}$, and we obtain

$$
\left.\mathcal{H}_{n+1}(z ; q, t) F_{n+1}(z)\right|_{z_{1}^{0} z_{2}^{0} \cdots z_{n+1}^{0}}=\left.\left.\sum_{T \in \mathcal{T}_{n}} \mathcal{H}_{n}(z ; q, t) F^{\prime}(z) \prod_{1 \leq k \leq n}\left(1-z_{k} w_{T}(k)\right)\right|_{S_{T}}\right|_{z_{k+1}^{0}} .
$$


Since every SYT on $\{0, \ldots, n+1\}$ is uniquely obtained from an SYT on $\{0, \ldots, n\}$ by adding $n+1$ to an outer corner, it is sufficient to show that for every $T \in \mathcal{T}_{n}$ we have

$$
\left.\left.\mathcal{H}_{n}(z ; q, t) F^{\prime}(z) \prod_{1 \leq k \leq n}\left(1-z_{k} w_{T}(k)\right)\right|_{S_{T}}\right|_{z_{n+1}^{0}}=\left.\sum_{T^{\prime}} \mathcal{H}_{n+1}(z ; q, t) F(z) \prod_{1 \leq k \leq n+1}\left(1-z_{n+1} w_{T^{\prime}}(n+1)\right)\right|_{S_{T^{\prime}}},
$$

where $T^{\prime}$ ranges over all SYTs obtained from $T$ by adding $n+1$ to an outer corner.

When taking constant term with respect to $z_{n+1}$, we only need to consider factors containing $z_{n+1}$, so we only need to work with the product

$$
\begin{aligned}
F_{n+1}(z) & \left.\frac{\left(1-z_{n+1}\right)\left(1-q t z_{n+1}\right)}{\left(1-q z_{n+1}\right)\left(1-t z_{n+1}\right)} \prod_{1 \leq k \leq n} \frac{\left(1-z_{n+1} / z_{k}\right)\left(1-q t z_{n+1} / z_{k}\right)}{\left.\left(1-q z_{n+1} / z_{k}\right)\left(1-t z_{n+1} / z_{k}\right)\right)}\right|_{S_{T}}= \\
& =\left.F_{n+1}(z)\right|_{S_{T}} \frac{\left(1-z_{n+1}\right)\left(1-q t z_{n+1}\right)}{\left(1-q z_{n+1}\right)\left(1-t z_{n+1}\right)} \prod_{1 \leq k \leq n} \frac{\left(1-z_{n+1} w_{T}(k)\right)\left(1-q t z_{n+1} w_{T}(k)\right)}{\left(1-q z_{n+1} w_{T}(k)\right)\left(1-t z_{n+1} w_{T}(k)\right)} .
\end{aligned}
$$

This is a proper rational function in $z_{n+1}$, since $F_{n+1}(z)$ contains only negative powers in $z_{n+1}$. But it is not clear which denominator factors are contributing. Indeed there are plenty of cancelations that are easier to describe using the Omega notation. Now, the key part of our product can be rewritten in the form

$$
\Omega\left[-M z_{n+1}\right] \prod_{1 \leq k \leq n} \Omega\left[-M z_{n+1} w_{T}(k)\right]=\Omega\left[-M z_{n+1}\left(1+w_{T}(1)+\cdots+w_{T}(n)\right)\right]=\Omega\left[-z_{n+1} M B_{T}\right],
$$

where $B_{T}$ is precisely as defined in 3.3. Now using 1.22 we may in turn rewrite this as

$$
\Omega\left[z_{n+1}\left(x_{0}+x_{1}+\cdots+x_{m}-u_{0}-u_{1}-\cdots-u_{m}\right]=\frac{\prod_{j=0}^{m}\left(1-z_{n+1} u_{j}\right)}{\prod_{j=0}^{m}\left(1-z_{n+1} x_{j}\right)},\right.
$$

where $u_{0}=1, x_{0}, x_{1}, \ldots, x_{m}$ and $u_{0}, u_{1}, \ldots, u_{m}$ are the corner weights of the shape of $T$. It results from this that the contributing factors in the denominators are $\left(1-x_{i} z_{n+1}\right)$ for $x_{i}$ the weight of an outer corner of $T$. For each such corner cell $c$ construct $T^{\prime}$ by adding $n+1$ to $T$ at the cell $c$. We thus obtain

$$
\left.\left.\mathcal{H}_{n}(z ; q, t) F^{\prime}(z) \prod_{1 \leq k \leq n}\left(1-z_{k} w_{T}(k)\right)\right|_{S_{T}}\right|_{z_{n+1}^{0}}=\left.\sum_{T^{\prime}} \mathcal{H}_{n+1}(z ; q, t) F_{n+1}(z) \prod_{1 \leq k \leq n+1}\left(1-z_{k} w_{T^{\prime}}(k)\right)\right|_{S_{T^{\prime}}}
$$

where $T^{\prime}$ ranges over all SYT's obtained from $T$ by adding $n+1$ to an outer corner.

This completes the proof.

We can clearly see that, by appropriately specializing $F_{n}(z), 3.21$ gives I.14. To see that it contains 3.15 as well we need only give the right hand side of 3.21 a non recursive construction. To this end, note that from the proof, we see that $\left(1-z_{k} w_{T}(k)\right)$ cancels with one factor in the denominator. This is $\Omega\left[z_{k} w_{T}(k)\right]$ and becomes $\Omega[1]$ after the substitution $z_{k}^{-1}=w_{T}(k)$. The same substitution also produces an "- 1 " to cancel this "1" within the Omega bracket. However $\Omega[1]$ itself has no meaning and the cancelation $\Omega[1-1]=1$ sometimes is not correct. For instance, we may have the following

$$
\left.\Omega\left[z-z^{3}\right]\right|_{z=1}=\left.\frac{1-z^{3}}{1-z}\right|_{z=1}=1+z+\left.z^{2}\right|_{z=1}=3
$$


This is not a problem but rather only another manifestation of the $0 / 0$ form in calculus limits. The general situation is guided by the following rule:

$$
\left.\Omega\left[z^{a_{1}}+\cdots+z^{a_{\ell}}-z^{b_{1}}-\cdots-z^{b_{\ell}}\right]\right|_{z=1}=\left.\prod_{i=1}^{\ell} \frac{\left(1-z^{b_{i}}\right) /(1-z)}{\left(1-z^{a_{i}}\right) /(1-z)}\right|_{z=1}=\frac{b_{1} \cdots b_{\ell}}{a_{1} \cdots a_{\ell}} .
$$

In our calculations, the $a_{i}$ and $b_{i}$ are all equal to 1 , so we can simply cancel them. With this provision the result of the recursive use of the substitution set $S_{T}$ can also be computed as follows

$$
\begin{aligned}
\left(-\frac{1}{M}\right)^{n} \prod_{1 \leq i \leq n} f\left(z_{i}\right) & \left.\prod_{1 \leq i>j \leq n} f\left(z_{j} / z_{i}\right) \prod_{1 \leq k \leq n}\left(1-z_{k} w_{T}(k)\right)\right|_{S_{T}}= \\
= & \left.\left(-\frac{1}{M}\right)^{n} \Omega\left[-M\left(z_{1}+\cdots+z_{n}\right)-M \sum_{1 \leq i<j \leq m} z_{j} / z_{i}-\sum_{1 \leq k \leq n} z_{k} w_{T}(k)\right]\right|_{S_{T}} \\
& =\left(-\frac{1}{M}\right)^{n} \Omega\left[-M \sum_{1 \leq k \leq n} w_{t}(k)^{-1}-M \sum_{1 \leq i<j \leq m} w_{T}(i) / w_{T}(j)-n\right]
\end{aligned}
$$

Using this expression for the summand in 3.21 we clearly see that 3.15 is also a special case of 3.21 .

As we mentioned in the introduction computer data suggests that the Tesler polynomial

$$
P_{a_{1}, a_{2}, \ldots, a_{n}}(q, t)=\left(-\frac{1}{M}\right)^{n} \sum_{A \in \mathcal{T}\left(a_{1}, a_{2}, \ldots, a_{n}\right)} w t(A)
$$

has non-negative integer coefficients when $a_{1} \leq a_{2} \leq \cdots \leq a_{n}$. Further computer explorations prompted by the identity

$$
P_{a_{1}, a_{2}, \ldots, a_{n}}(q, t)=(-1)^{n} M \partial_{p_{1}} \nabla^{-a_{n}} \partial_{p_{1}} \nabla^{a_{n}-a_{n-1}} \cdots \partial_{p_{1}} \nabla^{a_{3}-a_{2}} \partial_{p_{1}} \nabla^{a_{2}-a_{1}} \partial_{p_{1}} \nabla^{a_{1}} p_{n+1}\left[\frac{X}{M}\right]
$$

revealed an even stronger fact. Namely, starting with $p_{n+1}\left[\frac{X}{M}\right]$, repeated applications of the operator $\partial_{p_{1}} \nabla^{a}$ (with $a \geq 0$ ) invariably yields a Schur positive symmetric function. Thus it seems plausible that all these positivities may have a Representation Theoretical proof based on Haiman's discovery [7] of the role of the operator $\nabla$ in the Algebraic Geometry of the Hilbert Scheme. The authors have been trying to contact Mark Haiman regarding this matter, but so far without success.

At any rate it would be preferable to have a more elementary approach to proving these positivities. In the last few pages it will be instructive to see how this can be achieved by constant term methods.

To this end let us set

$$
\mathcal{K}\left(a_{1}, \ldots, a_{n}\right):=\left.\frac{1}{(-M)^{n} x_{1}^{a_{1}} \cdots x_{n}^{a_{n}}} \prod_{1 \leq i \leq n} f\left(x_{i}\right) \prod_{1 \leq i<j \leq n} f\left(x_{j} / x_{i}\right)\right|_{x^{0}} .
$$

This given, we can form the generating function

$$
G_{n}\left(y_{1}, \ldots, y_{n}\right)=\sum_{a_{i} \geq 0} \mathcal{K}\left(a_{1}+\cdots+a_{n}+1, a_{2}+\cdots+a_{n}+1, \ldots, a_{n}+1\right) y_{1}^{a_{1}} \cdots y_{n}^{a_{n}}
$$

and show that all coefficients of $G(y)$ in the $y_{i}^{\prime} s$ are polynomials in $\mathbf{N}[q, t]$. 
We can obtain a constant term expression for $G_{n}(y)$ as follows. It is easy to see that

$$
\sum_{a_{i} \geq 0} \frac{y_{1}^{a_{1}} \cdots y_{n}^{a_{n}}}{x_{1}^{a_{1}+\cdots+a_{n}+1} \cdots x_{n}^{a_{n}+1}}=\frac{x_{1}^{-1} \cdots x_{n}^{-1}}{\left(1-\frac{y_{1}}{x_{1}}\right)\left(1-\frac{y_{2}}{x_{1} x_{2}}\right) \cdots\left(1-\frac{y_{n}}{x_{1} x_{2} \cdots x_{n}}\right)} .
$$

Thus it follows that

$$
G_{n}(y)=\left.\mathcal{G}_{n}(y ; x)\right|_{x^{0}},
$$

where

$$
\mathcal{G}_{n}(y ; x)=\frac{x_{1}^{-1} \cdots x_{n}^{-1}}{\left(1-\frac{y_{1}}{x_{1}}\right)\left(1-\frac{y_{2}}{x_{1} x_{2}}\right) \cdots\left(1-\frac{y_{n}}{x_{1} x_{2} \cdots x_{n}}\right)} \frac{1}{(-M)^{n}} \prod_{1 \leq i \leq n} f\left(x_{i}\right) \prod_{1 \leq i<j \leq n} f\left(x_{j} / x_{i}\right) .
$$

Using this formula we can compute $G_{n}(y)$ for $n=1,2,3$ quickly using Xin's Ell2 package. For example,

$$
G_{2}(y)=\frac{1+t+q-q t y_{1}+q t^{2} y_{2}+q^{2} t y_{2}+q^{2} t^{2} y_{2}-q t^{3} y_{1} y_{2}-q^{2} t^{2} y_{1} y_{2}-q^{3} t y_{1} y_{2}-q^{2} t^{3} y_{1} y_{2}-q^{3} t^{2} y_{1} y_{2}}{\left(1-t y_{1}\right)\left(1-q y_{1}\right)\left(1-q t y_{2}\right)\left(1-q^{3} y_{2}\right)\left(1-t^{3} y_{2}\right)} .
$$

In this form, showing the positivity does not appear promising. The following lemma is straightforward, but significantly reduces the complexity.

\section{Lemma 3.1}

Suppose $F(q, t)=\sum_{i, j} c_{i, j} q^{i} t^{j}$ is a polynomial with integer coefficients. Then the positivity of

$$
\widetilde{F}(q, t)=\sum_{i>j}\left(c_{i, j}-c_{j, i}\right) q^{i} t^{j}
$$

implies the positivity of the divided difference

$$
\partial_{q, t} F(q, t)=\frac{F(q, t)-F(t, q)}{q-t}=\partial_{q, t} \widetilde{F}(q, t) \in \mathbf{N}[q, t] .
$$

Proof

Direct computation yields

$$
\partial_{q, t} q^{i} t^{j}= \begin{cases}q^{i-1} t^{j}+q^{i-2} t^{j+1}+\cdots+q^{j} t^{i-1} & \text { if } i>j, \\ 0, & \text { if } i=j, \\ -\partial_{q, t} q^{j} t^{i}=-\left(q^{j-1} t^{i}+q^{j-2} t^{i+1}+\cdots+q^{i} t^{j-1}\right), & \text { if } i<j .\end{cases}
$$

which clearly implies the lemma.

\section{Remark 3.2}

The lemma clearly extends by linearity for series with coefficients polynomials in $q, t$. Moreover, it is not hard to see that $\widetilde{F}(q, t)$ can be computed by

$$
\widetilde{F}(q, t)=\left.F(\alpha, \beta) \frac{1}{\left(1-\frac{q}{\alpha}\right)\left(1-\frac{q t}{\alpha \beta}\right)}\right|_{\alpha^{0} \beta^{0}}-\left.F(\alpha, \beta) \frac{1}{\left(1-\frac{q}{\beta}\right)\left(1-\frac{q t}{\alpha \beta}\right)}\right|_{\alpha^{0} \beta^{0}} .
$$

\section{Theorem 3.2}

The positivity conjecture holds for $n=1,2,3$. 


\section{Proof}

The $n=1$ case is trivial since $G_{1}\left(y_{1}\right)=\frac{y_{1}}{\left(1-q y_{1}\right)\left(1-t y_{1}\right)}$.

For $n \geq 2$ we can show the positivity as follows. We first take constant term in $x_{1}$. That is the constant term in $x_{1}$ of

$$
\frac{x_{1}^{-1} \cdots x_{n}^{-1}}{\left(1-\frac{y_{1}}{x_{1}}\right)\left(1-\frac{y_{2}}{x_{1} x_{2}}\right) \cdots\left(1-\frac{y_{n}}{x_{1} x_{2} \cdots x_{n}}\right)} \frac{\left(1-x_{1}\right)\left(1-q t x_{1}\right)}{\left(1-q x_{1}\right)\left(1-t x_{1}\right)} \prod_{j=2}^{n} \frac{\left(1-x_{j} / x_{1}\right)\left(1-q t x_{j} / x_{1}\right)}{\left(1-q x_{j} / x_{1}\right)\left(1-t x_{j} / x_{1}\right)} .
$$

Only the factors $\left(1-q x_{1}\right)$ and $\left(1-t x_{1}\right)$ are contributing, so we have

$$
\left.\mathcal{G}_{n}(y ; x)\right|_{x_{1}^{0}}=\left.\mathcal{G}_{n}(y ; x)\left(1-q x_{1}\right)\right|_{x_{1}=q^{-1}}+\left.\mathcal{G}_{n}(y ; x)\left(1-t x_{1}\right)\right|_{x_{1}=t^{-1}} .
$$

Now observe that $\mathcal{G}_{n}(y ; x)$ is symmetric in $q$ and $t$. Thus if we set

$$
F_{n}(y ; q, t)=\left.\left.(q-t) \mathcal{G}_{n}(y ; x)\left(1-q x_{1}\right)\right|_{x_{1}=q^{-1}}\right|_{x^{0}},
$$

then we shall have

$$
G_{n}(y)=\partial_{q, t} F_{n}(y ; q, t)=\frac{F_{n}(y ; q, t)-F_{n}(y ; t, q)}{q-t} .
$$

The Ell2 package delivers $F_{n}(y ; q, t)$ quickly for $n=2,3,4$. The $n=2$ case is simple:

$$
F_{2}(y ; q, t)=\frac{q(1+q)}{\left(1-q y_{1}\right)\left(1-q t y_{2}\right)\left(1-q^{3} y_{2}\right)} .
$$

Lemma 3.1 applies with $\widetilde{F}_{2}(y ; q, t)=F_{2}(y ; q, t)$ and the positivity of $G_{2}(y)$ follows. For the $n=3$ case, we obtain

$$
F_{3}(y ; q, t)=\frac{\text { (a lengthy polynomial) }}{\left(1-q y_{1}\right)\left(1-q t y_{2}\right)\left(1-q^{3} y_{2}\right)\left(1-q t^{3} y_{3}\right)\left(1-q^{2} t^{2} y_{3}\right)\left(1-q^{3} t y_{3}\right)\left(1-q^{6} y_{3}\right)} .
$$

We can use Remark 3.2 to compute $\widetilde{F}_{3}(y ; q, t)$. By letting $E_{3}=E_{3}(y ; q, t)=\widetilde{F}_{3}(y ; q, t / q)$, we have

$$
E_{3}(y ; q, t)=\frac{\text { (a lengthy polynomial) }}{\left(1-q y_{1}\right)\left(1-t y_{2}\right)\left(1-t^{2} y_{3}\right)\left(1+t^{2} y_{3}\right)\left(1-q^{2} t y_{3}\right)\left(1-q^{3} y_{2}\right)\left(1-t^{3} y_{1}^{2} y_{3}\right)\left(1-q^{6} y_{3}\right)} .
$$

The positivity of $G_{3}(y)$ clearly follows from that of $E_{3}$, which is obvious once we rewrite

$$
\begin{aligned}
E_{3}(y ; q, t)=\frac{(1+q)^{2} q^{2}}{\left(1-t y_{2}\right)\left(1-q y_{1}\right)\left(1-t^{2} y_{3}\right)\left(1-q^{2} t y_{3}\right)} \\
+\frac{y_{2} q^{4}\left(1+2 q+2 q^{2}+q^{3}\right)}{\left(1-t y_{2}\right)\left(1-q y_{1}\right)\left(1-t^{2} y_{3}\right)\left(1-q^{3} y_{2}\right)\left(1-q^{2} t y_{3}\right)} \\
\quad+\frac{y_{3} q^{4}\left(t+q^{3}\right)\left(1+2 q+2 q^{2}+q^{3}\right)}{\left(1-q y_{1}\right)\left(1-t^{2} y_{3}\right)\left(1-q^{3} y_{2}\right)\left(1-q^{2} t y_{3}\right)\left(1-q^{6} y_{3}\right)} \\
\quad+\frac{q\left(1+t+q+t y_{1}+q t^{2} y_{3}+t^{3} y_{1} y_{3}+q t^{2} y_{1} y_{3}+q t^{3} y_{1} y_{3}\right)}{\left(1-t y_{2}\right)\left(1-q y_{1}\right)\left(1-q^{2} t y_{3}\right)\left(1-t^{3} y_{1}{ }^{2} y_{3}\right)\left(1-t^{4} y_{3}^{2}\right)}
\end{aligned}
$$


The above form is obtained by using partial fraction decompositions and inspections. We first observe the positivity of $E_{3}(y ; q, t)\left(1-y_{1} q\right)\left(1-y_{3} q^{2} t\right)$, whose partial fraction with respect to $y_{1}$ is reasonably simple:

$$
E_{3}(y ; q, t)\left(1-y_{1} q\right)\left(1-y_{3} q^{2} t\right)=Q+\frac{q\left(1+t+q+t y_{1}+q t^{2} y_{3}+t^{3} y_{1} y_{3}+q t^{2} y_{1} y_{3}+q t^{3} y_{1} y_{3}\right)}{\left(1-t y_{2}\right)\left(1+t^{2} y_{3}\right)\left(1-t^{2} y_{3}\right)\left(1-t^{3} y_{1}^{2} y_{3}\right)},
$$

where $Q$ is a little complex and the second term is already positive. Now the partial fraction decomposition of $Q$ with respect to $y_{2}$ is

$$
Q=\frac{q^{2}\left(t+2 q t+q^{2}+q^{2} t+q^{3}\right)}{\left(1-t y_{2}\right)\left(1-t^{2} y_{3}\right)\left(t-q^{3}\right)}-\frac{q^{4}\left(1+2 q+2 q^{2}+q^{3}\right)}{\left(t-q^{3}\right)\left(1-q^{3} y_{2}\right)\left(1-q^{6} y_{3}\right)} .
$$

It is an easy exercise to obtain from the above the desired form.

Our method at the moment appears forbidding for $n=4$. The formula for $\widetilde{F}_{4}(y ; q, t)$ is already significantly complex.

\section{Bibliography}

[1] F. Bergeron and A. M. Garsia, Science fiction and Macdonald polynomials, Algebraic methods and q-special functions (Montreal, QC, 1996), CRM Proc. Lecture Notes, vol. 22, Amer. Math. Soc., Providence, RI, 1999, pp. 1-52.

[2] F. Bergeron, A. M. Garsia, M. Haiman, and G. Tesler, Identities and positivity conjectures for some remarkable operators in the theory of symmetric functions, Methods in Appl. Anal. 6 (1999), 363-420.

[3] A.M. Garsia and M. Haiman, A remarkable q,t-Catalan sequence and q-Lagrange inversion, J. Algebraic Combin. 5 (1996), no. 3, 191-244.

[4] A. Garsia, M. Haiman and G. Tesler, Explicit Plethystic Formulas for the Macdonald q,t-Kostka Coefficients, Séminaire Lotharingien de Combinatoire, B42m (1999), 45 pp.

[5] A. Garsia, Combinatorics of Tesler matrices in the Theory of Parking Functions and Diagonal Harmonics, (Lecture notes)

[6] J. Haglund A polynomial expression for the Hilbert series of the quotient ring of diagonal coinvariants, Adv. Math., to appear.

[7] M. Haiman, Hilbert schemes, polygraphs, and the Macdonald positivity conjecture, J. Amer. Math. Soc. 14 (2001), 941-1006.

[8] I. G. Macdonald, Symmetric functions and Hall polynomials, Oxford Mathematical Monographs, second ed., Oxford Science Publications, The Clarendon Press Oxford University Press, New York, 1995.

[9] G. Xin, The ring of Malcev-Neumann series and the residue theorem, PhD. Dissertation at Brandeis University (May 2004).

[10] G. Xin, A fast algorithm for MacMahon's partition analysis, Electron. J. Combin., 11 (2004), R58. (electronic)

[11] M. Zabrocki, UCSD Advancement to Candidacy Lecture Notes, Posted in "http : //www.math.ucsd.edu/ garsia/somepapers/". 